\title{
Chalcone Synthase Transcripts Are Detected in Alfalfa Root Hairs Following Inoculation with Wild-Type Rhizobium meliloti
}

\author{
Heather I. McKhann, ${ }^{1}$ Nancy L. Paiva, ${ }^{3}$ Richard A. Dixon, ${ }^{3}$ and Ann M. Hirsch ${ }^{1,2}$ \\ ${ }^{1}$ Department of Molecular, Cell and Developmental Biology and ${ }^{2}$ Molecular Biology Institute, University of \\ California, Los Angeles, Los Angeles, CA 90095-1606 U.S.A.; ${ }^{3}$ Plant Biology Division, The Samuel Rob- \\ erts Noble Foundation, Ardmore, OK 73402 U.S.A. \\ Received 19 April 1996. Accepted 21 October 1996.
}

Flavonoids are involved in a number of critical events in the interaction between nitrogen-fixing bacteria and legumes. To get a better understanding of the importance of flavonoids in the earliest stages of the alfalfa-Rhizobium meliloti symbiosis, we followed the expression of two chalcone synthase (CHS) gene family members as well as of chalcone isomerase (CHI) and isoflavone reductase (IFR) genes. CHS transcripts increased 2 to 4 dpi (days postinoculation) with wild-type rhizobia, but not after inoculation with the heterologous $R$. leguminosarum bv. trifolii or with an exopolysaccharide (exo) mutant of $R$. meliloti. CHS transcripts were detected in the root hairs and epidermal cells of the root hair zone, and infrequently in nodule primordia. Insignificant CHI and IFR mRNA accumulation over control levels was observed in response to rhizobial inoculation. The slight increase in CHS transcript accumulation following wild-type $R$. meliloti inoculation was correlated with an observed increase in root flavonoid content as well as a change in the nod gene-inducing activity of the root exudate. The nod gene-inducing flavonoids exuded from wild-type rhizobia-inoculated roots were identified as $4^{\prime}$, 7dihydroxyflavone and 4, 4' dihydroxy-2'-methoxychalcone. Although there was a slight increase over the uninoculated controls in the level of medicarpin-3-O $O$-glucoside $6^{\prime \prime}-O$ malonate (MGM) in extracts of roots inoculated with rhizobia, IFR transcript accumulation was not significantly elevated over that of the controls. Moreover, no medicarpin aglycone was detected in the inoculated roots. Thus, although inoculation with wild-type rhizobia triggers some of the genes induced during an interaction between a host and a pathogen, the expression of these genes in the Rhizobium-legume interaction is at a very low level, suggesting that rhizobia have evolved a mechanism(s) to avoid triggering the host's defense responses.

During the symbiotic interaction between Rhizobium (sensu lato) and leguminous plants, a series of signaling events that

Corresponding author: A. M. Hirsch; E-mail: hirsch@biovxi.biology.ucla.edu

Present address of H. I. McKhann: Department of Molecular Cell Biology, University of Utrecht, Padualaan 8, $3584 \mathrm{CH}$ Utrecht, The Netherlands culminate in the formation of a nitrogen-fixing nodule takes place. Flavonoids exuded from legume seeds and roots induce rhizobial nodulation (nod) genes (Mulligan and Long 1985; Kosslak et al. 1987; Firmin et al. 1986; Maxwell et al. 1989; Peters et al. 1986; Redmond et al. 1986). Nod factor, lipochitooligosaccharides synthesized by the products of the nod genes, is responsible for some of the earliest events in the symbiosis, including root hair curling (Lerouge et al. 1990), cortical cell division (Truchet et al. 1991), preinfection thread formation (van Brussel et al. 1992), and expression of some early nodulin genes (Vijn et al. 1993, 1995; Horvath et al. 1993; Journet et al. 1993; Bauer et al. 1994; Crespi et al. 1994; Y. Fang and A. M. Hirsch, unpublished results).

Flavonoids have been implicated in many other aspects of the Rhizobium-legume symbiosis besides induction of nod genes. For example, flavonoids have been proposed to be involved in nodule morphogenesis (Hirsch 1992; Yang et al. 1992). Another postulated role for flavonoids in the rhizobialegume interaction is as part of a host defense reaction in response to the pathogenic or parasitic nature of Rhizobium (see reviews by Vance 1978; Djordjevic et al. 1987; Long and Staskawicz 1993; McKhann and Hirsch 1994a). Isoflavonoids are precursors of phytoalexins which are synthesized as part of the hypersensitive response to incompatible pathogens (see Dixon 1986). These compounds, as well as other phenolics, have been shown to accumulate in some ineffective symbioses, suggesting that the host legume mounts a defense response to the invading Fix ${ }^{-}$rhizobia (Werner et al. 1985; Parniske et al. 1991, 1994; Yang et al. 1992; Grosskopf et al. 1993; Vasse et al. 1993).

In this study, we have investigated alfalfa's response to $R$. meliloti inoculation by monitoring the expression of CHS (chalcone synthase), CHI (chalcone isomerase) and IFR (isoflavone reductase) genes, the genes which encode enzymes of the phenylpropanoid biosynthetic or "defense" pathway. CHS is the enzyme involved in the first step committed to flavonoid biosynthesis, the synthesis of the chalcone. This reaction is followed by a ring closure, mediated by $\mathrm{CHI}$, to produce a flavanone. IFR is a later step in the pathway; it catalyzes the penultimate step in medicarpin biosynthesis (Paiva et al. 1991). By using cDNA clones of two of the four CHS genes that we previously determined to be expressed in nitrogen-fixing nodules (McKhann and Hirsch 
1994b), we show that the nodule-expressed CHS4-1 mRNAs are slightly elevated in alfalfa roots that were inoculated with wild-type rhizobia compared to uninoculated roots. In addition, CHS6-4 transcripts are detected in epidermal and root hair cells of inoculated roots. The early increase in CHS transcript accumulation is correlated with an increased production of nod-gene inducing flavonoids. However, neither CHI nor IFR mRNA levels are increased in $R$. meliloti-inoculated roots compared to uninoculated controls. Moreover, no medicarpin was detected in alfalfa roots that had been inoculated with either wild-type or exo mutant $R$. meliloti. This suggests that rhizobia employ some sort of mechanism to avoid triggering a potential host defense response.

\section{RESULTS}

Nodule-enhanced chalcone synthase gene family members are expressed following inoculation with wild-type and mutant $R$. meliloti.

Four cDNA clones, CHS6-4, CHS4-1, CHS12-1, and CHS4-2, had been isolated from an alfalfa nodule cDNA library; thus, they are likely to represent CHS mRNAs that are expressed in the symbiotic interaction (McKhann and Hirsch 1994b). We used RNAse protection analysis to show that CHS transcripts accumulated in alfalfa root tissue following $R$. meliloti inoculation. Probes CHS4-1P and CHS6-4P were made from CHS4-1 and CHS6-4 and are 175 and 148 bp, respectively, DNA fragments generated by PCR and subcloned into the vector pGEM4Z (McKhann and Hirsch 1994b). Both probes had been determined previously to give specific protected fragments in RNAse protection analysis (McKhann 1993; McKhann and Hirsch 1994b).

We first determined the level of CHS4-1 and CHS6-4 transcripts in uninoculated roots grown in the presence of $20 \mathrm{mM}$ nitrate. Uninoculated roots grown in the absence of nitrate

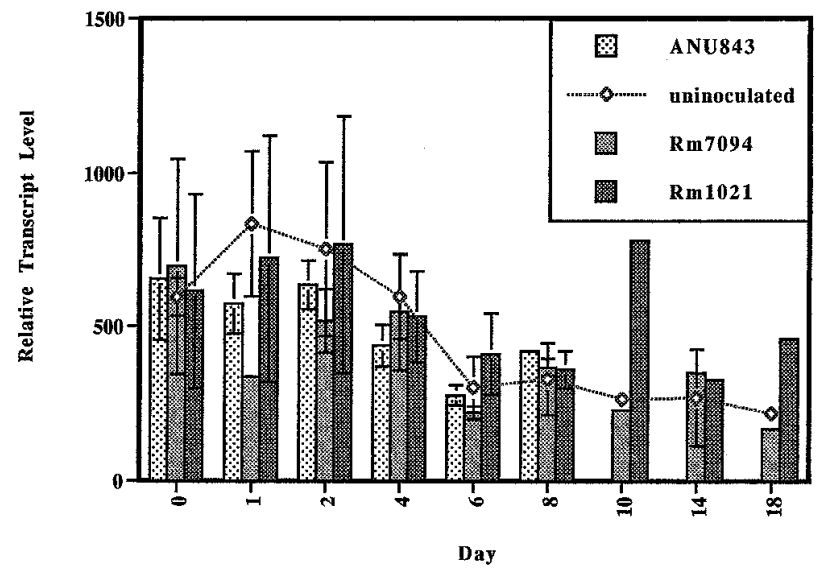

Fig. 1. RNAse protection assays comparing the temporal expression of CHS6-4 in uninoculated roots grown in the presence of $20 \mathrm{mM}$ nitrate (line graph) and roots inoculated with heterologous (ANU843; lightshaded bars) and homologous (Rm7094 and Rm1021; darker shaded bars) rhizobia. Samples were taken over the 18-day experimental period except for ANU843, which was sampled until day 8 only. The transcript levels are normalized to the amount of Msc27-hybridizing RNA present in each sample. The line above and below the average values indicates the standard deviation. If absent, the standard deviations were too small to be displayed. were previously shown to contain low levels of CHS4-1 and CHS6-4 mRNA (McKhann and Hirsch 1994b). Nitrate was added to ensure that the plants remained healthy and that flavonoids were not synthesized as part of a stress response. We used a heterologous Rhizobium (R. leguminosarum bv. trifolii; ANU843) and an exopolysaccharide (exo) $R$. meliloti mutant (Rm7094) as additional controls to show that rhizobial inoculation per se did not trigger CHS gene expression (Figs. 1 and 2). CHS transcript levels were followed for only 8 dpi with ANU843, but for the complete 18 day-period following Rm7094 inoculation. In both cases, the amount of CHS transcript accumulation did not increase significantly over that of the uninoculated plus nitrate control.

RNAse protection analysis was then used to determine whether CHS transcripts accumulated in wild-type $R$. melilotiinoculated alfalfa roots compared to the control roots. An average value plus standard deviation at a specific time point for three independent experiments for CHS6-4 (Fig. 1) and for two independent experiments for CHS4-1 (Fig. 2) is presented here.

For the CHS6-4 gene, there was little difference in relative transcript levels between RNAs isolated from total root systems inoculated with wild-type $R$. meliloti or left uninoculated until $10 \mathrm{dpi}$, at which time there was an approximately threefold increase in CHS6-4 transcript levels in Rm1021inoculated roots (Fig. 1). In contrast, even as early as 2 dpi, there was an obvious increase (nearly 100\%) in CHS4-1 transcript accumulation. CHS4-1 transcript levels appeared to decrease relative to the uninoculated control levels at 4 dpi. However, this may be in part due to more CHS4-1 transcripts accumulating in the uninoculated control roots in contrast to the ANU843- and Rm7094-inoculated roots. At 6 dpi, the level of CHS4-1 transcripts in Rm1021-inoculated roots was significantly elevated over that of the controls and remained so for the duration of the experiment (Fig. 2).

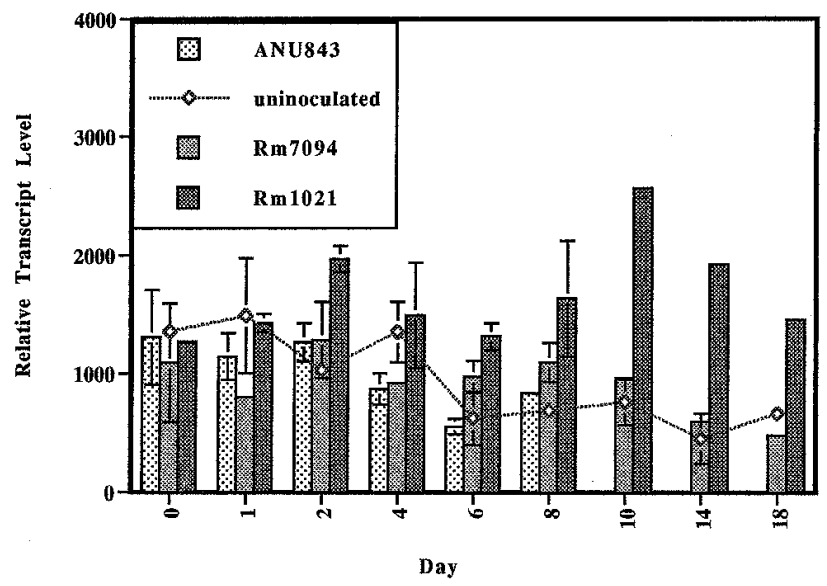

Fig. 2. RNAse protection assays comparing the temporal expression of CHS4-1 in uninoculated roots grown in the presence of $20 \mathrm{mM}$ nitrate (line graph) and roots inoculated with heterologous (ANU843; lightshaded bars) and homologous (Rm7094 and Rm1021; darker shaded bars) rhizobia. Samples were taken over the 18-day experimental period except for ANU843, which was sampled until day 8 only. The transcript levels are normalized to the amount of Msc27-hybridizing RNA present in each sample. The line above and below the average values indicates the standard deviation. If absent, the standard deviations were too small to be displayed. 


\section{Spatial patterns of CHS expression 1 to 10 days after inoculation}

We chose to study CHS6-4 by in situ hybridization analysis because CHS6-4 transcripts accumulate in uninoculated control roots to lower levels than CHS4-1 mRNAs (compare Yaxes in Figs. 1 and 2). CHS transcripts corresponding to the gene CHS6-4 were first localized by in situ hybridization in Rm1021-inoculated roots 10 dpi using the spot inoculation technique (data not shown); 10 days was the treatment time when the highest level of CHS6-4 transcripts was detected in inoculated roots using RNAse protection methods. Transcripts were not detectable in transverse (Fig. 3A) or in longitudinal sections (data not shown) of roots that had been shaminoculated.

We then proceeded backwards in time to look at roots which had been exposed to Rm1021 for fewer than 10 days. Examination of roots 2 to 6 dpi revealed a high density of silver grains in epidermal and emergent root hair cells adjacent to the site of spot inoculation (Fig. 3B-C). The silver grains correspond to transcripts that hybridized to the CHS6-4 probe. In some roots, the cells of vascular bundle of inoculated plants (Fig. 3C) or the endodermal cells of lateral roots (Fig. 3H) were also refractile. Occasionally, silver grains were associated with phloem fibers. No signal above background was detected in sections from inoculated roots that had been hybridized with a sense probe (data not shown).

Nodule primordia were also examined for CHS transcripts. The earliest time point when we could detect nodule primordia in spot inocculated roots was $3 \mathrm{dpi}$, but silver grains indicating the presence of CHS transcripts were not observed in these cells. Occasionally, signal was detected in nodule primordia cells $4 \mathrm{dpi}$, but the silver grains were aggregated and associated with starch grains (arrows, Fig. 3D, E). In most nodule primordia and developing nodules, the strongest bona fide signal was detected in root hair (left arrow) and epidermal cells surrounding the pre-emergent primordium (Fig. 3E). However, we detected silver grains over inner cortical cell derivatives of nodule primordia formed 4 dpi in two of 20 young nodules examined (Fig. 3F, G). The photographic emulsion had been left to expose for 12 weeks for these nodules. In contrast to the aggregated appearance as seen in Figure $3 \mathrm{E}$, the silver grains in Figure $3 \mathrm{G}$ were evenly distributed over the cytoplasm of the nodule primordium cells. Surprisingly, cells which contained infection threads did not exhibit significantly higher levels of CHS6-4 transcripts than noninfected cells (arrows, Fig. 3F, G).
CHS6-4-hybridizing transcripts were also not detected within the cells of lateral root primordia, which are clearly distinguishable from nodule primordia. On the other hand, parent root cells surrounding young lateral roots often contained significant amounts of CHS transcripts (arrows, Fig. $3 \mathrm{H}, \mathrm{I})$. A similar localization has been cited for transgenic tobacco plants containing a bean CHS promoter-GUS construct (Schmid et al. 1990).

\section{Expression of CHI and IFR.}

Northern analysis was used to measure the levels of CHI and IFR gene expression. In uninoculated roots grown without nitrate, CHI transcripts levels were relatively constant and present at relatively low levels throughout the 18-day experimental period (see McKhann and Hirsch 1994b). For uninoculated roots grown in the presence of $20 \mathrm{mM}$ nitrate for 18 days or in roots inoculated with exo mutants and grown under nitrogen-limiting conditions for the same time period, CHI transcript levels were low (Fig. 4). When alfalfa roots were inoculated with wild-type $R$. meliloti, no increase in CHI transcript levels was detected (Fig. 4). Indeed, starting at 6 $\mathrm{dpi}$, there was a statistically significant decrease in CHI transcript accumulation in Rm1021-inoculated roots compared to both uninoculated and Rm7094-inoculated roots.

The levels of IFR transcripts over the 18-day experimental period exhibited a pattern similar to that of CHI (Fig. 5). None of the treatments were statistically different from one another or from the day 0 uninoculated control. There was an almost $50 \%$ increase in IFR transcript levels in Rm1021-inoculated roots 2 dpi over the uninoculated control levels, but the large standard deviation makes this increase appear insignificant.

\section{Increase in nod-gene inducing flavonoids.}

To determine whether the increased accumulation of CHS transcripts in response to wild-type $R$. meliloti could be correlated with the Ini response, we examined root exudate from

Table 1. $\beta$-Glactosidase activity (in Miller units) expressed by nodABC::lacZ fusions in response to exudates from uninoculated roots and roots inoculated with $\mathrm{Rm} 1021$

\begin{tabular}{lccc}
\hline Sample & Trial 1 & Trial 2 & Trial 3 \\
\hline Jensen's Medium & 15.8 & 5.9 & 38.7 \\
Exudate - Rm1021 & 16.5 & 10.9 & 35.3 \\
Exudate + Rm1021 & 33.3 & 32.6 & 56.5 \\
Fold Induction & 2.0 & 3.0 & 1.6 \\
\hline
\end{tabular}

Fig. 3. In situ hybridization analysis of wild-type Rhizobium meliloti spot-inoculated roots using probes corresponding to CHS6-4P (see McKhann and Hirsch 1994b). A, Dark-field photomicrograph of a cross section of a sham-inoculated root 4 dpi. No silver grains (detected as white spots) are observed over background. Exposed for 4 weeks. Bar, $50 \mu \mathrm{m}$. B, Dark-field photomicrograph of a cross section of a root 2 dpi. CHS transcripts (detected as white spots) are located mostly in the root hairs (arrows). Exposed for 4 weeks. Bar, $50 \mu \mathrm{m}$. C, Dark-field photomicrograph of a cross-section of root 6 dpi near the point of spot-inoculation. Most of the signal is in the root hairs (arrows). The stele is highly refractile because silver grains are deposited on fiber cells. Exposed for 12 weeks. Bar, $50 \mu \mathrm{m}$. D, Bright-field photomicrograph of the region in E (arrow) that shows light scattering. Silver grains, shown as a dark spots, bind non-specifically to the starch grains in these cells (arrow). Bar, $15 \mu \mathrm{m}$. E, Dark-field photomicrograph (non-median section) of a cross section of root with a nodule primordium 4 dpi with $R$. meliloti. Exposed for 4 weeks. The right arrow points to starch grains. Silver grains are also associated with the outer regions of the nodule and the root hairs (left arrow). Bar, $45 \mu \mathrm{m}$. F, Bright-field photomicrograph of a cross section of root with a nodule primordium 4 dpi. The interior cells of the nodule primordium are covered with silver grains. The arrow points to an infection thread. Exposed for 12 weeks. Bar, $50 \mu \mathrm{m}$. G, Dark-field photomicrograph of (F). Silver grains are deposited in the cells of the nodule primordium. The arrow points to an infection thread. H, Dark-field photomicrograph of a lateral root. Silver grains overlay the epidermal and cortical cells of the parent root (arrow). Some light scattering is also observed in the endodermis. Exposed for 4 weeks. Bar, $40 \mu \mathrm{m}$. I, Bright-field photomicrograph of the same lateral root. Bar, $40 \mu \mathrm{m}$. 

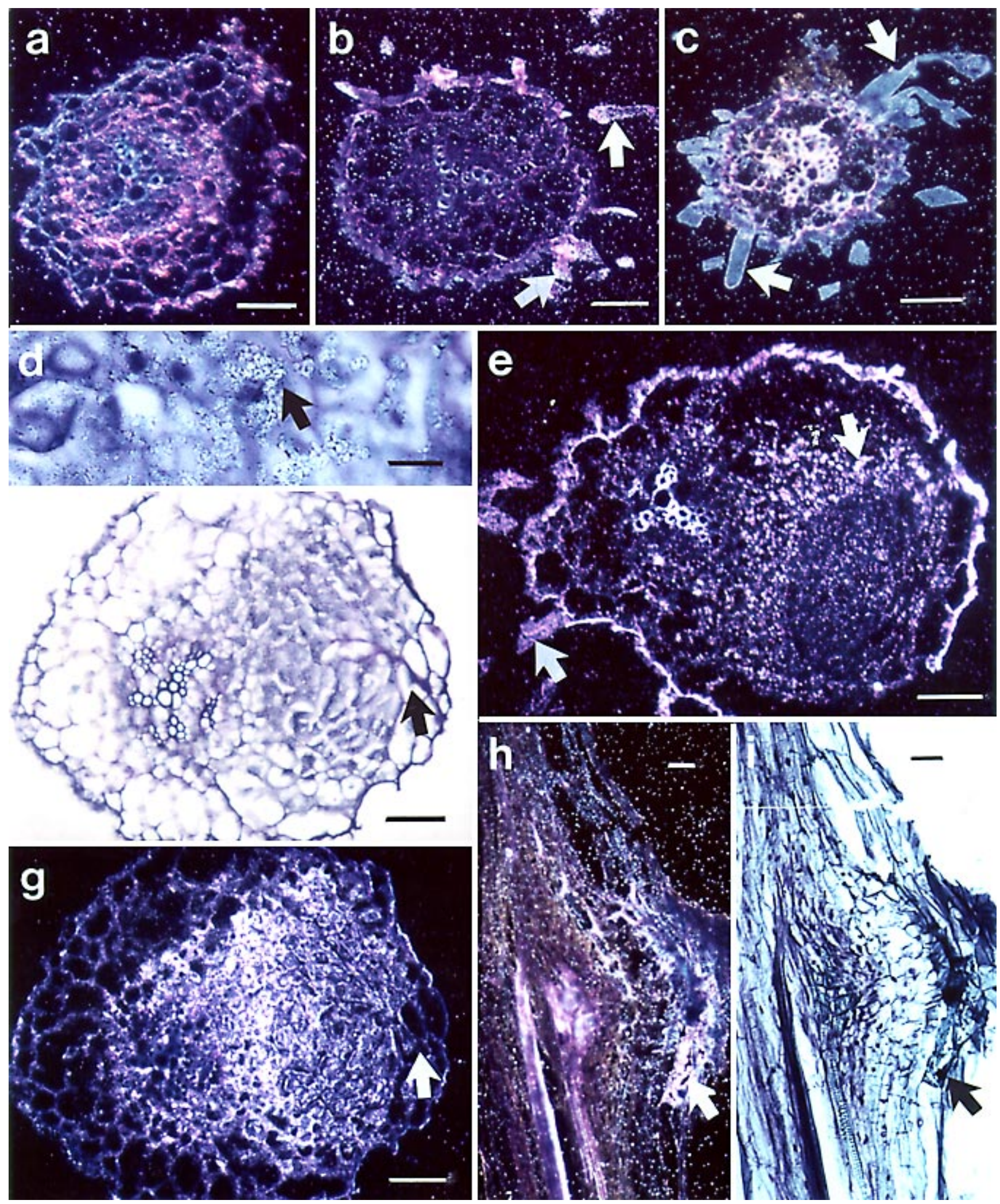
inoculated plants and uninoculated plants 5 dpi. In three separate experiments, an average twofold increase in nod gene induction was measured in exudates following inoculation with wild-type $R$. meliloti (Table 1).

Examination of the flavonoid profile of root exudates confirmed that higher levels of flavonoids known to induce rhizobial nod genes are found in exudates from roots inoculated with wild-type $R$. meliloti (Fig. 6A). The largest increase was in 4', 7-dihydroxyflavone $(30.9 \mathrm{ng} / \mathrm{ml}$ compared to $0 \mathrm{ng} / \mathrm{ml}$ for exudates from uninoculated roots). A fourfold increase in 4, 4'-dihydroxy-2'-methoxychalcone was also detected (Fig. 6A). In contrast, no 4', 7-dihydroxyflavone or 4, 4'dihydroxy-2'-methoxychalcone was detected in exudates prepared from roots inoculated with the $R$. meliloti exo mutant (Fig. 6A). A small increase in glycosylated formononetin as well as in two unknown compounds was also observed (data not shown). The concentrations of these flavonoids, however, were very low, making it difficult to determine any statistical differences among the three treatments.

A small increase was observed in flavonoid content in extracts made from roots inoculated with wild-type or exoB $R$. meliloti in comparison to extracts from uninoculated roots (Fig. 6B). Analysis of the flavonoid profile of inoculated roots at $5 \mathrm{dpi}$ indicated that the level of formononetin 7-Oglucoside-6"- $O$-malonate (FGM) is approximately $40 \%$ higher than that in uninoculated root extracts (Fig. 6B). An increase of approximately $110 \%$ in medicarpin-3- $O$-glucoside6"- $O$-malonate (MGM) was also observed in extracts prepared from R. meliloti-exoB mutant inoculated roots and an increase of $75 \%$ in MGM in wild-type $R$. meliloti-inoculated roots over the uninoculated controls. In a second experiment, the levels of FGM and MGM in wild-type $R$. melilotiinoculated roots were higher but more similar to those in uninoculated roots, and the levels of both FGM and MGM were significantly higher in $R$. meliloti exoB mutant inoculated roots than in the uninoculated roots (60 to $70 \%$ increases) (data not shown). Despite the increases in MGM, no increase in free medicarpin (medicarpin aglycone) was observed. MGM is thought to be a non-toxic storage form of medicarpin which is sequestered in the vacuole, whereas medicarpin aglycone is the active antimicrobial form (Paiva et al. 1991; Oommen et al. 1994). Low levels of the nod gene inducers 7, 4'-dihydroxyflavone or 4, 4'-dihydroxy-2'methoxychalcone were found in extracts derived from $R$. meliloti-exoB-inoculated roots (approximately $1.2 \mu \mathrm{g} / \mathrm{g}$ and $0.6 \mu \mathrm{g} / \mathrm{g}$, respectively), very similar to the levels found in extracts from uninoculated and wild-type $R$. meliloti-inoculated roots.

\section{DISCUSSION}

We have examined the spatial and temporal patterns of expression for the CHS6-4 gene and the temporal patterns of CHS, CHI, and IFR genes during the establishment of the Rhizobium meliloti-alfalfa symbiosis. The CHS clones used in this study were two of four that had been previously found in a nodule cDNA library (McKhann and Hirsch 1994b). Because CHS4-1 and CHS6-4 are 94.2\% homologous along their entire length, we used RNAse protection analysis to follow specifically the levels of transcripts corresponding to the two nodule-expressed CHS gene family members.
CHS, but not CHI and IFR transcripts, were shown to accumulate within 2 dpi in wild-type $R$. meliloti-inoculated roots. The lack of elevated CHS6-4 transcript levels observed in the Rm1021-inoculated roots using RNAse protection as opposed to in situ hybridization may result from the fact that for the RNAse protection assays, RNA from total root systems was used. Thus, CHS6-4 transcripts present in total root tissue would be very dilute. Consistent with this hypothesis is the observation based on in situ hybridization experiments showing that CHS6-4 transcripts accumulate in very few root cells.

The in situ hybridization experiments also demonstrated that CHS6-4 mRNAs accumulated in several distinct places in alfalfa roots inoculated with wild-type $R$. meliloti: the root hairs/epidermis, phloem fiber cells, and the nodule primordium, although we did not detect CHS6-4 transcripts routinely in the latter. The presence of silver grains in meristematic cells of the nodule primordium correlated with longer exposures times. This may have allowed the detection of small amounts of CHS6-4 mRNA in the tissues or its crosshybridization to other CHS gene family member mRNAs. The latter explanation seems less likely because our probe was a very small DNA fragment that is specific to one CHS gene family member-CHS6-4.

The epidermal and root hair sites of CHS gene expression are consistent with a potential Ini (increased nod gene induction) response. Schmelzer et al. (1988) had previously detected CHS mRNA, CHS protein, and CHS biosynthetic products in epidermal cells of parsley, suggesting that flavonoids are synthesized and secreted from the epidermis. The increased levels of CHS transcripts in roots inoculated with wild-type $R$. meliloti may correlate with the observed increase in nod gene-inducing flavonoids in root exudates. 4', 7dihydroxyflavone and 4, 4'-dihydroxy-2'-methoxychalcone, both known nod gene inducers, were detected in exudates obtained from wild-type $R$. meliloti-inoculated roots. Following $\beta$-glucosidase treatment of the exudate, some formononetin was detected, indicating that formononetin glycosides were also present in the root exudate. Our results are thus consistent with those of Dakora et al. (1993) who also measured an increase in formononetin-7- $O$-glucoside-6"- $O$-malonate in root exudates of inoculated plants. However, Dakora et al. (1993) also observed upon inoculation a large increase in medicarpin and medicarpin-3-O-glycoside, compounds which do not induce rhizobial nod genes. They also saw a small decrease in dihydroxyflavone, a moderate nod-gene inducer, which we did not observe in uninoculated roots. The reason for the disparity in the two sets of experiments is unknown, but different cultivars (Moapa 69 vs. Iroquois), seed sterilization procedures, and culture conditions were used. Our results are more similar to those of Recourt et al. (1992) who found nod gene-inducing flavonoids and no phytoalexins in vetch root exudates, and then only after inoculation with effective rhizobia. Moreover, based on the low level of IFR transcript accumulation 0 to 4 $\mathrm{dpi}$, the synthesis of high levels of medicarpin-3-O-glucoside during the early stages of nodulation seems unlikely.

FGM, MGM, coumestrol, and daidzein accumulate in roots also during the early stages of the VAM fungus-Medicago interaction (Harrison and Dixon 1993). In addition, 4',7dihydroxyflavone was found to accumulate in alfalfa mycorrhizal roots after colonization, and CHS transcript levels 
increased approximately 2-fold in the VAM-infected root early in the interaction. These results are similar to what we observed following wild-type $R$. meliloti inoculation. However, differences were also found. Harrison and Dixon (1993) measured a decrease in IFR mRNA levels, whereas we detected no such decrease in IFR transcript levels following wild-type $R$. meliloti inoculation. Also, in contrast to the VAM situation, where a transient increase in free medicarpin occurs, we did not detect free medicarpin in roots inoculated with either wild-type or exoB mutant $R$. meliloti.

Interestingly, nod gene inducing flavonoids were not detected in exudates prepared from roots 5 dpi with the $R$. meliloti exoB mutant nor were CHS, CHI, and IFR transcript

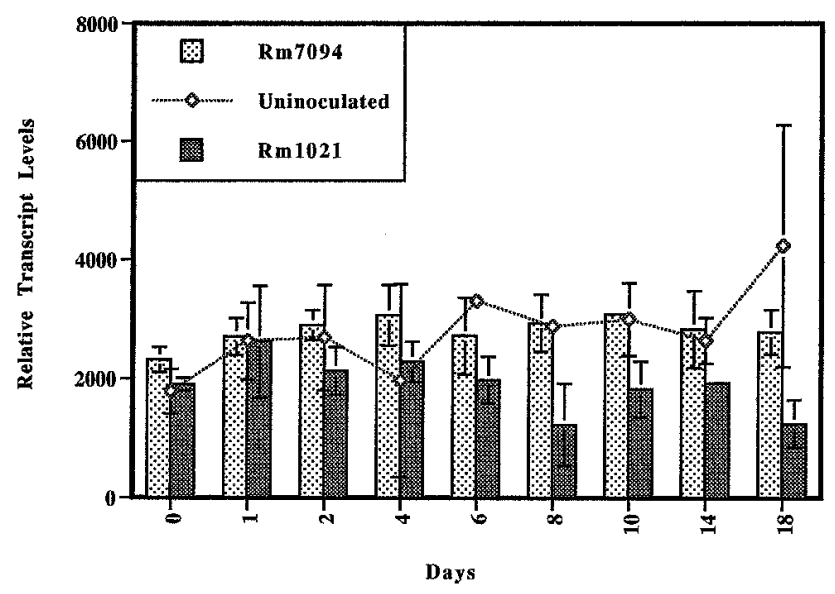

Fig. 4. Relative CHI gene transcript levels using Northern blot analysis for inoculated versus uninoculated roots grown in the presence of 20 $\mathrm{mM}$ nitrate from two independent experiments. The line above and below the average values indicates the standard deviation. If absent, the standard deviations were too small to be displayed. The values for the uninoculated roots are given as a line graph. The light-shaded bars are the values for Rm7094-inoculated roots and the dark-shaded bars are for Rm1021-inoculated roots. The CHI transcript levels are normalized to the amount of Msc27-hybridizing RNA in each sample.

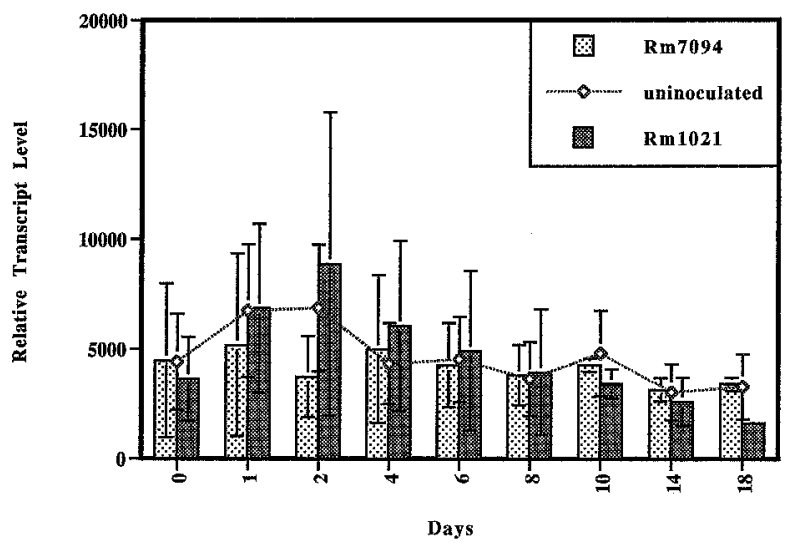

Fig. 5. Relative IFR gene transcript levels using Northern blot analysis for inoculated versus uninoculated roots grown in the presence of 20 $\mathrm{mM}$ nitrate from two independent experiments. The line above and below the average value indicates the standard deviation. If absent, the standard deviations were too small to be displayed. The values for the uninoculated roots are given as a line graph. The light-shaded bars are the values for Rm7094-inoculated roots and the dark-shaded bars are for Rm1021-inoculated roots. The IFR transcript levels are normalized to the amount of Msc27-hybridizing RNA in each sample. levels significantly higher in roots inoculated with Rm7094 compared to wild-type $R$. meliloti-inoculated roots. This suggests that the plant is specifically responding to wild-type $R$. meliloti 2 to 6 dpi to produce nod-gene inducing flavonoids, and, moreover, it is not responding to exo mutant rhizobia in the same way. Thus, even at this early stage of the nodule development pathway, the plant can discriminate an ineffective strain from an effective one. Extracts from $R$. meliloti wildtype and $e x o B$-inoculated roots showed an increase in FGM and MGM levels over the extracts from uninoculated roots, but the levels of FGM and MGM in R. meliloti exo mutant
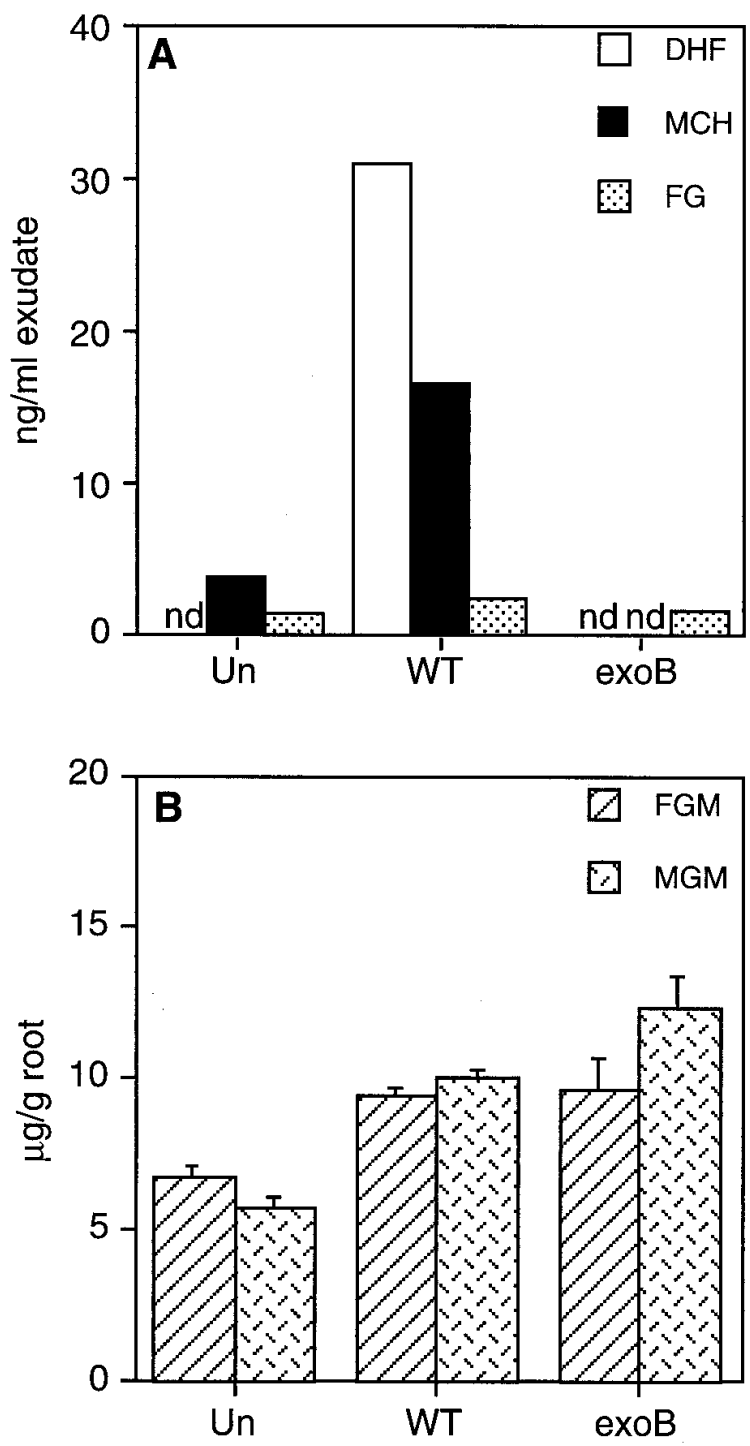

Fig. 6. Flavonoid composition of (A) root exudates and (B) root extracts. Plants were grown as described in the Materials and Methods and the roots were left uninoculated (Un) or were inoculated with wild-type Rhizobium meliloti-(WT) Rm1021 or with the R. meliloti exopolysaccharide mutant Rm7094 (exoB). Root exudates and extracts of root tissues collected 5 days after the start of the experiment were subjected to HPLC analysis before and after $\beta$-glucosidase treatment. DHF, 7, 4'dihydroxyflavone; $\mathrm{MCH}, 4$, 4'-dihydroxy-2'-methoxychalcone; FG, glycosides of formononetin estimated by amount of free formononetin following $\beta$-glucosidase treatment; FGM, formononetin-7- $O$-glucoside6 "'- $O$-malonate; MGM, medicarpin-3-O-glucoside-6" $O$-malonate. 
and wild-type-inoculated root extracts were low compared to what would be present in extracts from tissues or cell cultures infected with a pathogen or elicitor (Paiva et al. 1991). Estabrook and Sengupta-Gopalan (1991) demonstrated that symbiotic-specific PAL and CHS genes, which are clearly distinct from other gene family members induced by stress or pathogens, are activated during the early stages of the soybeanBradyrhizobium japonicum symbiosis. Thus, it seems unlikely that the plant is responding to $R$. meliloti, even to the ineffective exo mutant strain, as it would to a potential pathogen that triggers a hypersensitive response. Furthermore, it would appear that either that a host-defense response is suppressed, or that ineffective rhizobia are not recognized by the plant in the same way that incompatible pathogens are. Similarly, Harrison and Dixon (1993) proposed that there is a suppression of certain defense transcripts in the VAM symbiosis. Although there is an initial elevation of defense gene transcript levels, it is not maintained and is insufficient to prevent further colonization by the fungus.

In this vein, Mithöfer et al. (1996) have shown that cyclic 1,3-1,6 $\beta$-glucans from Bradyrhizobium japonicum USDA110 are inactive in stimulating phytoalexin synthesis, which is normally elicited by 1,3-1,6 $\beta$-glucans derived from the fungal pathogen Phytophthora sojae on its host soybean. Moreover, the bacterial $\beta$-glucans were shown to be ligands for the $\beta$ glucan-binding sites, the putative receptors for this elicitor in soybean roots, and thus were proposed to be suppressors of the $P$. sojae $\beta$-glucans. When a cyclic glucan from $R$. meliloti was tested on soybean, this $1,2-\beta$-glucan was inactive as elicitor, and also as a ligand of the $\beta$-glucan binding sites (Mithöfer et al. 1996). However, it would be of interest to see whether $R$. meliloti oligosaccharides interfere with fungal $\beta$ glucan activity when alfalfa is infected with a host-specific pathogen. Based on our results, we predict that such a response should take place.

\section{MATERIALS AND METHODS}

\section{Growth conditions.}

Medicago sativa L. cv. Iroquois seeds were sterilized as described in Löbler and Hirsch (1993). The seedlings were either grown in plastic dish pans containing vermiculite and perlite as previously described (Hirsch et al. 1989), or in dish pans on wire mesh covered with cheesecloth, suspended over Jensen's medium (Löbler and Hirsch 1993). Three days after germination, the seedlings were inoculated with wild-type (Rm1021; Meade et al. 1982), or exoB mutant (Rm7094; Finan 1988) Rhizobium meliloti strains. Total root systems were harvested at the specified time intervals and frozen immediately in liquid nitrogen. $R$. meliloti cultures, grown in RDM (Rhizobium Defined Medium; Vincent 1970) to an $\mathrm{OD}_{600}$ of 0.2 to 0.5 , were used to inoculate plants, after the bacteria had been washed with sterile water.

\section{Spot inoculations.}

For spot inoculations, seeds were sterilized as described above and placed on top of $1.5 \%$ nitrogen-free Jensen's agar in square petri dishes (LabTek). Seeds were allowed to germinate in the dark and after $72 \mathrm{~h}$, the seedlings were inoculated with 1 to $2 \mathrm{nl}$ of $R$. meliloti strains Rm1021 or Rm7094 as described by Dudley et al. (1987). Control plants were sham- inoculated with RDM or water alone, and India ink was used to mark the point of inoculation. Root hair deformation and nodulation were monitored by light microscopy. At the times indicated, plants were harvested. Approximately $5 \mathrm{~mm}$ of the root adjacent to the spot were harvested and fixed in formaldehyde-alcohol-acetic acid (FAA) for in situ hybridization (McKhann and Hirsch 1993).

\section{In situ hybridizations.}

In situ hybridizations were performed as described by McKhann and Hirsch (1993) except that ${ }^{33}$ P-UTP was used. Hybridizations were performed at $45^{\circ} \mathrm{C}$ in $50 \%$ formamide using CHS6-4P antisense and sense probes. Twenty inoculated roots from 1 to $10 \mathrm{dpi}$ and 10 uninoculated roots were prepared and examined. Over 21 specimens of nodule primordia ranging in developmental age from 3 to $10 \mathrm{dpi}$, and 20 different mature nodules ( $>21 \mathrm{dpi}$ ) were examined. After the in situ hybridization procedure, the slides were placed in the dark and the photographic emulsion was developed after times ranging from 4 to 12 weeks. Photographs were taken on a Zeiss Axiophot microscope using Ektachrome Tungsten 160 film.

\section{RNA isolation and Northern analysis.}

Total RNA was isolated from roots as described previously (McKhann and Hirsch 1994b). RNA was subjected to electrophoresis on a formaldehyde gel (Sambrook et al. 1989), and blotted onto Nytran following the instructions of Schleicher \& Schuell. Each lane was loaded with $10 \mu \mathrm{g}$ of RNA. Restriction fragments used as probes were labeled by random priming using $\alpha-{ }^{32} \mathrm{P}-\mathrm{dCTP}(\mathrm{NEN})$. For the $\mathrm{CHI}$ probe, the entire EcoRI insert of CHI-1 was used (McKhann and Hirsch 1994b). The IFR cDNA clone is a 1,181-bp EcoRI fragment (Paiva et al. 1991). The Msc27 clone (Kapros et al. 1992), used to monitor RNA loading, was obtained from D. Dudits (Hungarian Academy of Sciences, Szeged, Hungary) and is a 710-bp PstI fragment. The autoradiograms were quantified using a PhosphorImager (Molecular Dynamics).

Representative Northern blots were chosen from two or more independent experiments to be illustrated in the figures. The graphs were generated using Cricket Graph III (Computer Associates). To compensate for variations in experiments done at different times and with different batches of radioactive precursors, the values obtained for the inoculated roots were normalized to the uninoculated root values by finding the difference between control and experimental treatments at time 0 and then using this value to offset the variation between experimentals and controls.

\section{RNAse protection studies.}

RNAse protection was performed as described previously (McKhann and Hirsch 1994b). Riboprobes were generated from CHS4-1P (175 nt), CHS6-4P (148 nt), and Msc27P (105 nt) (McKhann and Hirsch 1994b). Approximately $1.2 \times 10^{6}$ cpm of probe RNA were added to each sample. The three probes, CHS4-1P, CHS6-4P, and Msc27P were in the same reaction mixture. Hybridizations were done at $42^{\circ} \mathrm{C}$ and in $50 \%$ formamide. A PhosphorImager (Molecular Dynamics) was used to quantify transcript levels. The transcript levels were corrected for loading differences by expressing the transcript level as the ratio of CHS4-1 or CHS6-4 to Msc27 (relative transcript level). All RNAse protections were done 
with RNA samples from two or more independent experiments. The transcript levels were averaged for each treatment and the standard deviations calculated using Cricket Graph III (Computer Associates). To ensure consistency, the values obtained for the inoculated plants were normalized to the relative transcript levels of the uninoculated controls as described above.

\section{Quantification of nod gene-inducing activity.}

To obtain root exudate, plants were grown in plastic pans on screens suspended over nitrogen-free Jensen's medium as described previously. Three-day-old seedlings were inoculated with either $R$. meliloti strain 1021, or left uninoculated. At 5 dpi, the root exudate was collected. The Rm1021-inoculated root exudates were centrifuged to remove bacterial cells and then filtered through an Acrodisc HT Tuffryn filter (Gelman Sciences). Total nod gene-inducing activity was determined using Rm1021/pRmM57 which contains a nodABC::lacZ fusion as described by Mulligan and Long (1985). The experiment was repeated three times.

\section{Flavonoid analysis of root exudates and root extracts.}

Analysis of flavonoids in roots and root exudates was performed as described previously (Paiva et al. 1991; Kessmann et al. 1991; Edwards and Kessmann 1992). Extracts and exudates were obtained from both wild-type and exoB mutant $R$. meliloti-inoculated roots. Extracts were analyzed by HPLC both directly and following $\beta$-glucosidase treatment to provide information on the content of both free and conjugated flavonoids. The identities of compounds were confirmed by a comparison of retention time and UV spectra (Beckman Model 168 diode array detector), and shifts in retention time following $\beta$-glucosidase treatments. Two different sets of exudates and extracts were examined.

\section{ACKNOWLEDGMENTS}

We thank D. Dudits (Szeged, Hungary) for Msc27 and Agway Inc. (Syracuse, NY) for supplying us with seeds of M. sativa cv. Iroquois. Yiwen Fang and James Shieh helped with quantifying some of the northern blots. Jacob S. Seeler and Richard Gaynor (Dallas, TX) are thanked for their help with the RNAse protections. We are grateful to the members of our laboratory for their helpful comments on the manuscript. Special thanks to Margaret Kowalczyk for preparing the color illustration. HIM was supported by a USDA training fellowship and a California Biotechnology training fellowship. A grant from the USDA (CRGO 91-37307-6603) to AMH also funded this research.

\section{LITERATURE CITED}

Bauer, P., Crespi, M. D., Szecsi, J., Allison, L. A., Schultze, M., Ratet, P., and Kondorosi, E. 1994. Alfalfa Enod12 genes are differentially regulated during nodule development by Nod factors and Rhizobium invasion. Plant Physiol. 105:585-592.

Crespi, M. D., Jurkevitch, E., Poiret, M., d'Aubenton-Carafa, Y., Petrovics, G., Kondorosi, E., and Kondorosi, A. 1994. enod40, a gene expressed during nodule organogenesis, codes for a non-translatable RNA involved in plant growth. EMBO J. 13:5099-5112.

Dakora, F. D., Joseph, C. M., and Phillips, D. A. 1993. Alfalfa (Medicago sativa L.) root exudates contain isoflavonoids in the presence of Rhizobium meliloti. Plant Physiol. 101:819-824.

Dixon, R. A. 1986. The phytoalexin response: Elicitation, signaling, and control of host gene expression. Biol. Rev. 61:239-291.

Djordjevic, M. A., Gabriel, D. W., and Rolfe, B. G. 1987. RhizobiumThe refined parasite of legumes. Annu. Rev. Phytopathol. 25:145-168.

Dudley, M. E., Jacobs, T. W., and Long, S. R. 1987. Microscopic studies of cell divisions induced in alfalfa roots by Rhizobium meliloti. Planta 171:289-301.

Edwards, R., and Kessmann, H. 1992. Isoflavonoid phytoalexins and their biosynthetic enzymes. Pages 45-62 in: Molecular Plant Pathology. A Practical Approach. Volume II. S. J. Gurr, M. J. MacPherson, and D. J. Bowles, eds. Oxford University Press, Oxford.

Estabrook, E. M., and Sengupta-Gopalan, C. 1991. Differential expression of phenylalanine ammonia-lyase and chalcone synthase during soybean nodule development. Plant Cell 3:299-308.

Finan, T. 1988. Genetic and physical analysis of Group E exo mutants of Rhizobium meliloti. J. Bacteriol. 170:474-477.

Firmin, J. L., Wilson, K. E., Rossen, L., and Johnston, A. W. B. 1986. Flavonoid activation of nodulation genes in Rhizobium reversed by other compounds present in plants. Nature 324:90-92

Grosskopf, E., Ha, D. T. C., Wingender, R., Röhrig, H., Szecsi, J., Kondorosi, E., Schell, J., and Kondorosi, A. 1993. Enhanced levels of chalcone synthase in alfalfa nodules induced by a Fix ${ }^{-}$mutant of Rhizobium meliloti. Mol. Plant-Microbe Interact. 6:173-181.

Harrison, M. J., and Dixon, R. A. 1993. Isoflavonoid accumulation and expression of defense gene transcripts during the establishment of vesicular-arbuscular mycorrhizal associations in roots of Medicago truncatula. Mol. Plant-Microbe Interact. 6:643-654.

Hirsch, A. M. 1992. Tansley Review No. 40. Developmental biology of legume nodulation. New Phytol. 122:211-237.

Hirsch, A. M., Bhuvaneswari, T. V., Torrey, J. G., and Bisseling, T. 1989. Early nodulin genes are induced in alfalfa root outgrowths elicited by auxin transport inhibitors. Proc. Natl. Acad. Sci. USA 86:1244-1248.

Horvath, B., Heidstra, R., Lados, M., Moerman, M., Spaink, H. P., Promé, J.-C., van Kammen, A., and Bisseling, T. 1993. Lipooligosaccharides of Rhizobium induce infection-related early nodulin gene expression in pea root hairs. Plant J. 4:727-733.

Journet, E. P., Pichon, M., Dedieu, A., de Billy, F., Truchet,. G., and Barker, D. G. 1994. Rhizobium meliloti Nod factors elicit cell-specific transcription of the ENOD12 gene in transgenic alfalfa. Plant J. 6:241-249.

Kapros, T., Bogre, L., Nemeth, K., Bako, L., Györgyey, J., Wu, S. C., and Dudits, D. 1992. Differential expression of histone H3 gene variants during cell cycle and somatic embryogenesis in alfalfa. Plant Physiol. 98:621-625.

Kessmann, H., Edwards, R., Geno, P. W., and Dixon, R. A. 1990. Stress responses in alfalfa (Medicago sativa L.) V. Constitutive and elicitorinduced accumulation of isoflavonoid conjugates in cell suspension cultures. Plant Physiol. 94:227-232.

Kosslak, R. M., Bookland, R., Barklei, J., Paaren, H. E., and Appelbaum, E. R. 1987. Induction of Bradyrhizobium japonicum common nod genes by isoflavones isolated from Glycine max. Proc. Natl. Acad. Sci. USA 84:7428-7432.

Lerouge, P., Roche, P., Faucher, C., Maillet, F., Truchet, G., Promé, J.C., and Dénarié, J. 1990. Symbiotic host-specificity of Rhizobium meliloti is determined by a sulphated and acylated glucosamine oligosaccharide signal. Nature 344:781-784.

Löbler, M., and Hirsch, A. M. 1993. A gene that encodes a proline-rich nodulin with limited homology to PsENOD12 is expressed in the invasion zone of Rhizobium meliloti-induced alfalfa root nodules. Plant Physiol. 103:21-30.

Long, S. R., and Staskawicz, B. J. 1993. Prokaryotic plant parasites. Cell 73:921-935.

Maxwell, C. A., Hartwig, U. A., Joseph, C. M., and Phillips, D. A. 1989. A chalcone and two related flavonoids released from alfalfa roots induce nod genes of Rhizobium meliloti. Plant Physiol. 91:842-847.

McKhann, H. I. 1993. The roles of flavonoids in the nodulation of alfalfa by Rhizobium meliloti: The effect of $R$. meliloti on chalcone synthase and chalcone isomerase gene expression patterns. Ph.D. Dissertation. University of California, Los Angeles.

McKhann, H. I., and Hirsch, A. M. 1993. In situ localization of specific mRNAs in plant tissues. Pages 179-205 in: Methods in Plant Molecular Biology and Biotechnology. B. R. Glick and J. E. Thompson, eds. CRC Press, Inc., Boca Raton, FL.

McKhann, H. I., and Hirsch, A. M. 1994a. Does Rhizobium avoid the host response? Pages 139-162 in: Current Topics in Microbiology and Immunology. Vol. 192. Bacterial Pathogenesis of Plants and Animals. J. Dangl, ed. Springer Verlag, Berlin.

McKhann, H. I., and Hirsch, A. M. 1994b. Isolation of chalcone syn- 
thase and chalcone isomerase cDNAs from alfalfa (Medicago sativa L.): Highest transcript levels occur in young roots and root tips. Plant Mol. Biol. 24:767-777

Meade, H. M., Long, S. R., Ruvkun, G. B., Brown, S. E., and Ausubel, F. M. 1982. Physical and genetic characterization of symbiotic and auxotrophic mutants of Rhizobium meliloti induced by transposon Tn5 mutagenesis. J. Bacteriol. 149:114-122.

Mithöfer, A., Bhagwat, A. A., Feger, M., and Ebel, J. 1996. Suppression of fungal $\beta$-glucan-induced plant defence in soybean (Glycine max L.) by cyclic 1,3-1,6- $\beta$-glucans from the symbiont Bradyrhizobium japonicum. Planta 199:270-275.

Mulligan, J. T., and Long, S. R. 1985. Induction of Rhizobium meliloti nodC expression by plant exudate required nodD. Proc. Natl. Acad. Sci. USA 82:6609-6613.

Oommen, A., Dixon, R. A., and Paiva, N. L. 1994. The elicitorinducible alfalfa isoflavone reductase promoter confers different patterns of developmental expression in homologous and heterologous transgenic plants. Plant Cell 6:1789-1803.

Paiva, N. L., Edwards, R., Sun, Y., Hrazdina, G., and Dixon, R. A. 1991. Stress responses in alfalfa (Medicago sativa L.). 11. Molecular cloning and expression of alfalfa isoflavone reductase, a key enzyme of isoflavonoid phytoalexin biosynthesis. Plant Mol. Biol. 17:653-667.

Parniske, M., Fischer, H.-M., Hennecke, H., and Werner, D. 1991. Accumulation of the phytoalexin glyceollin I in soybean nodules in fected by a Bradyrhizobium japonicum nifA mutant. Z. Naturforsch. 46c:318-320.

Parniske, M., Schmidt, P. E., Kosch, K., and Müller, P. 1994. Plant defense responses of host plants with determinate nodules induced by EPS-defective exoB mutants of Bradyrhizobium japonicum. Mol. Plant-Microbe Interact. 7:631-638.

Peters, N. K., Frost, J. W., and Long, S. R. 1986. A plant flavone, luteolin, induces expression of Rhizobium meliloti nodulation genes. Science 233:977-980.

Recourt, K., Schripsema, J., Kijne, J. W., van Brussel, A. A. N., and Lugtenberg, B. J. J. 1991. Inoculation of Vicia sativa subsp. nigra roots with Rhizobium leguminosarum biovar viciae results in release of nod gene activating flavanones and chalcones. Plant Mol. Biol. 16:841-852.

Recourt, K., van Tunen, A. J., Mur, L. A., van Brussel, A. A. N., Lugtenberg, B. J. J., and Kijne, J. W. 1992. Activation of flavonoid biosynthesis in roots of Vicia sativa subsp. nigra plants by inoculation with Rhizobium leguminosarum biovar viciae. Plant Mol. Biol. 19:411-420.

Redmond, J. W., Batley, M., Djordjevic, M. A., Innes, R. W., Kuempel, P. L., and Rolfe, B. G. 1986. Flavones induce expression of nodula- tion genes in Rhizobium. Nature 323:632-635.

Sambrook, J., Fritsch, E. F., and Maniatis, T. 1988. Molecular Cloning: A Laboratory Manual. 2nd Ed. Cold Spring Harbor Laboratory Press, Cold Spring Harbor, NY.

Schmid, J., Doerner, P. W., Clouse, S. D., Dixon, R. A., and Lamb, C. J. 1990. Developmental and environmental regulation of a bean chalcone synthase promoter in transgenic tobacco. Plant Cell 2:619-631.

Schmelzer, E., Jahnen, W., and Hahlbrock, H. 1988. In situ localization of light-induced chalcone synthase mRNA, chalcone synthase, and flavonoid end products in epidermal cells of parsley leaves. Proc. Natl. Acad. Sci. USA 85:2989-2993.

Truchet, G., Roche, P., Lerouge, P., Vasse, J., Camut, S., de Billy, F., Promé, J. C., and Dénarié, J. 1991. Sulphated lipooligosaccharide signals of Rhizobium meliloti elicit root nodule organogenesis in alfalfa. Nature 351:670-673.

van Brussel, A. A. N., Bakuizen, R., van Spronsen, P. C., Spaink, H. P., Tak, T., Lugtenberg, B. J. J., and Kijne, J. W. 1992. Induction of preinfection thread structures in the leguminous host plant by mitogenic lipooligosaccharides of Rhizobium. Science 257:70-72.

van Brussel, A. A. N., Recourt, K., Pees, E., Spaink, H. P., Tak, T., Wijffelman, C. A., Kijne, J. W., and Lugtenberg, B. J. J. 1990. A biovar-specific signal of Rhizobium leguminosarum bv. viciae induces increased nodulation gene-inducing activity in root exudate of Vicia sativa subsp. nigra. J. Bacteriol. 172:5394-5401.

Vance, C. P. 1983. Rhizobium infection and nodulation: A beneficial plant disease? Annu. Rev. Microbiol. 37:399-424.

Vasse, J., de Billy, F., and Truchet, G. 1993. Abortion of infection during the Rhizobium meliloti-alfalfa symbiotic interaction is accompanied by a hypersensitive reaction. Plant J. 4:555-566.

Vijn, I., das Neves, L., van Kammen, A., Franssen, H., and Bisseling, T. 1993. Nod factors and nodulation in plants. Science 260:1764-1765.

Vijn, I., Martinez-Abaraca, F., Yang, W. C., das Neves, L., van Brussel, A., van Kammen, A., and Bisseling. T. 1995. Early nodulin gene expression during Nod factor-induced processes in Vicia sativa. Plant $\mathbf{J}$. 8:111-119.

Vincent, J. M. 1970. A Manual for the Practical Study of Root-Nodule Bacteria. Blackwell Scientific Publications, London.

Werner, D., Mellor, R. B., Hahn, M. G., and Grisebach,. H. 1985. Soybean root response to symbiotic infection. Glyceollin I accumulation in an ineffective type of soybean nodule with an early loss of peribacteroid membrane. Z. Naturforsch. 40c:179-181.

Yang, W.-C., Cremers, H. C. J. C., Hogendijk, P., Katinakis, P., Wijffelman, C. A., Franssen, H., van Kammen, A., and Bisseling, T. 1992. In-situ localization of chalcone synthase mRNA in pea root nodule development. Plant J. 2:143-151. 